\title{
Raman Scattering from ZnSe Nanolayers
}

\author{
D. Nesheva ${ }^{a, *}$, M.J. Šćepanovićc ${ }^{b}$, S. AšKrabić ${ }^{b}$, Z. Levi ${ }^{a}$, I. Bineva ${ }^{a}$ And Z.V. Popović ${ }^{b}$ \\ ${ }^{a}$ Institute of Solid State Physics, Bulgarian Academy of Sciences \\ 72 Tzarigradsko Chaussee Blvd, 1784 Sofia, Bulgaria \\ ${ }^{b}$ Center for Solid State Physics and New Materials, Institute of Physics \\ Pregrevica 118, Belgrade 11080, Serbia
}

\begin{abstract}
A series of ZnSe single layers having thickness between $30 \mathrm{~nm}$ and $1 \mu \mathrm{m}$ was deposited on c-Si and glass substrates at room substrate temperature. Thermal evaporation of ZnSe powder in high vacuum has been applied. Moreover, $\mathrm{SiO}_{x} / \mathrm{ZnSe}$ periodic multilayers prepared by the same deposition technique and having ZnSe layer thickness of 2 and $4 \mathrm{~nm}$ have been studied. Raman spectra were measured at $295 \mathrm{~K}$, using the $442 \mathrm{~nm}$ line of a $\mathrm{He}-\mathrm{Cd}$ laser as well as different lines of the $\mathrm{Ar}^{+}$or $\mathrm{Ar}^{+} / \mathrm{Kr}^{+}$lasers. The observed Raman features have been related to multiple optical phonon ( $1 \mathrm{LO}$ to $4 \mathrm{LO}$ ) light scattering and connected with the existence of randomly oriented crystalline ZnSe grains in both ZnSe single layers and ZnSe layers of the multilayers. Relatively large line width $\left(\approx 15 \mathrm{~cm}^{-1}\right)$ of the $1 \mathrm{LO}$ band has been observed and related to lattice distortion in the crystalline grains and existence of amorphous phase in the layers thinner than $100 \mathrm{~nm}$. The Raman spectra measured on both $\mathrm{ZnSe}$ single layers and $\mathrm{SiO}_{x} / \mathrm{ZnSe}$ multilayers using the $488 \mathrm{~nm}$ line with a gradually increased laser beam power indicate an increased crystallinity at high irradiation levels.
\end{abstract}

PACS numbers: 81.15.Ef, 81.05.Dz, 78.30.-j, 68.55.-a

\section{Introduction}

The interest in ZnSe low-dimensional structures is caused by the possibility of creation of effective light-emitting devices with spontaneous or coherent radiation in the green-blue part of the visible spectrum [1-3] or white light emission [4]. Besides, thin nanostructured semiconductor films, in particular II-VI ones, are promising materials for gas sensor applications ([5] and references therein), since in such layers the sensing surface is significantly larger than the geometric one. An in-depth understanding of the phonon propagation is of great technological importance for possible applications, not only in designing semiconductor light emitting and other optoelectronic devices but also in fast electronic devices.

In this study Raman scattering investigations are carried out on a series of ZnSe single layers and two kinds of periodic $\mathrm{SiO}_{x} / \mathrm{ZnSe}$ multilayers (MLs) deposited at room substrate temperature by thermal evaporation of ZnSe powder and glassy $\mathrm{SiO}$ in vacuum. It is shown that as-deposited single layers contain two phases, amorphous and crystalline. Increased film crystallinity is achieved by high power laser irradiation.

\section{Experimental details}

Single layers of ZnSe having thickness of 30, 40, 50, $70,100 \mathrm{~nm}$ and $1 \mu \mathrm{m}$ were deposited on crystalline

\footnotetext{
* corresponding author; e-mail: nesheva@issp.bas.bg
}

c-Si or Corning 7059 glass substrates held at $25^{\circ} \mathrm{C}$. The layers were produced by physical evaporation of powdered ZnSe (Merck, Suprapure) at a residual pressure of $\approx 3 \times 10^{-4} \mathrm{~Pa}$ from a tantalum crucible located in the bottom of a cylindrical screen (not intentionally heated). The top of the screen is close to the substrates; thus evaporation in a quasi-closed volume is carried out. A preliminarily calibrated quartz microbalance system was used to control the nominal layer thickness and deposition rate $\left(V_{\mathrm{d}}=1.5 \mathrm{~nm} / \mathrm{s}\right)$. Step-by-step deposition was carried out during which the substrates were rotated at a rate of 8 turns/min spending only $1 / 12$ part of the turn time over the ZnSe source. Thus, more than 30 "sublayers", with a nominal thickness of $\approx 1.0 \mathrm{~nm}$ in each step, form the respective layer.

Multilayers of $\mathrm{ZnSe} / \mathrm{SiO}_{x}(x \approx 1.7$ [6] $)$ were prepared by consecutive thermal evaporation of $\mathrm{ZnSe}$ $\left(V_{\mathrm{d}}=1.5 \mathrm{~nm} / \mathrm{s}\right)$ and glassy $\mathrm{SiO}\left(V_{\mathrm{d}}=0.2 \mathrm{~nm} / \mathrm{s}\right)$ from two independent tantalum crucibles at a vacuum of $10^{-3} \mathrm{~Pa}$ on Corning 7059 glass or crystalline $p$-Si substrates maintained at room temperature. The step-by-step procedure was applied in the deposition of each layer in the MLs, as well. The thickness of the ZnSe layers was 2.0 or $4.0 \mathrm{~nm}$, whereas that of the $\mathrm{SiO}_{x}$ layers was 2.5 or $4.0 \mathrm{~nm}$, respectively. This procedure allowed the fabrication of nanocrystalline/amorphous MLs with smooth interfaces and good periodicity [7].

Raman measurements were performed in the quasi-backscattering geometry using the $457.9 \mathrm{~nm}(2.71 \mathrm{eV})$, $488 \mathrm{~nm}(2.54 \mathrm{eV})$ or $514.5 \mathrm{~nm}(2.41 \mathrm{eV})$ line of an $\mathrm{Ar}^{+}$ laser or the $442 \mathrm{~nm}(2.8 \mathrm{eV})$ line of a $\mathrm{He}-\mathrm{Cd}$ laser as an 
excitation source, a Jobin-Yvon U1000 monochromator and a photomultiplier as a detector. Micro-Raman measurements were performed using a Jobin-Yvon T64000 triple spectrometer system, equipped with confocal microscope and a nitrogen-cooled CCD detector. The $488 \mathrm{~nm}$ line of a mixed $\mathrm{Ar}^{+} / \mathrm{Kr}^{+}$laser with spot diameter at the sample surface of about $50 \mu \mathrm{m}$ was used. All measurements were performed at room temperature in air. All spectra plotted on a same graph are scaled identically.

\section{Results and discussion}

Figure 1a shows three Raman spectra of a ZnSe layer with the thickness of $1 \mu \mathrm{m}$ deposited on a Corning 7059 glass substrate. We interpret the series of up to 4 peaks (at $\approx 250,500,750,1000 \mathrm{~cm}^{-1}$ ) as being due to the so-called longitudinal optical LO-phonon replicas in ZnSe, well known in polar semiconductors [8]. The replicas are better resolved at excitation conditions close to resonance Raman scattering conditions. The strongest enhancement of the Raman signal is observed when the exciting light approaches the optical band gap $E_{\mathrm{g}}^{0}$ of the material. As the $1 \mathrm{LO}$ band is the most intensive in the Raman spectrum excited by the $457.9 \mathrm{~nm}$ line, the energy of this line should be close to the optical band gap, although 4 replicas exist also in the spectrum obtained with $488 \mathrm{~nm}$. This is in agreement with previous results which have shown [9] that optical absorption obeys the law for direct allowed electronic transitions in crystalline semiconductors with a value of $E_{\mathrm{g}}^{0}=2.67 \mathrm{eV}$ for the optical band gap of $1 \mu \mathrm{m}$ thick ZnSe layer.

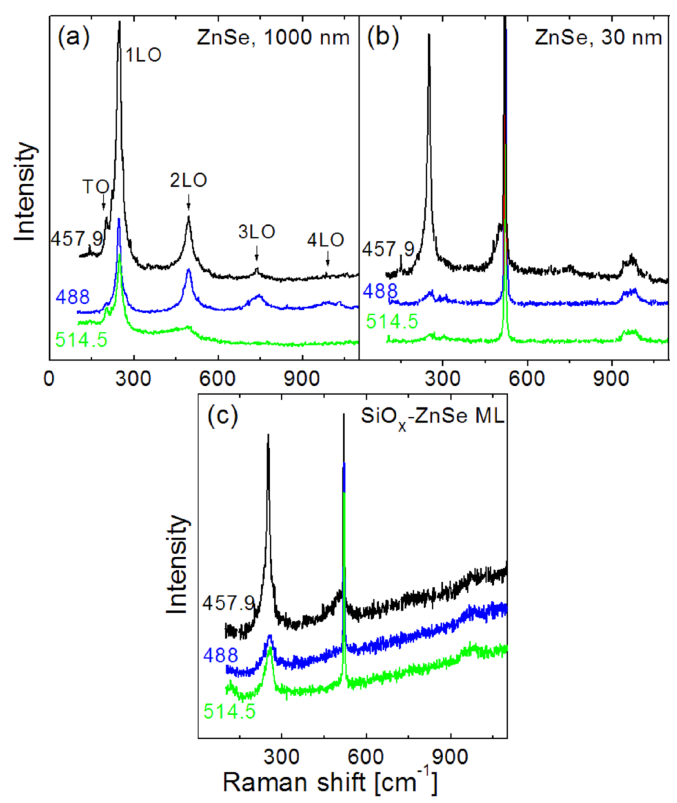

Fig. 1. Raman spectra of ZnSe single layers having thicknesses $1 \mu \mathrm{m}$ (a) and $30 \mathrm{~nm} \mathrm{(b),} \mathrm{and} \mathrm{a}$ $\mathrm{SiO}_{x}(4 \mathrm{~nm}) / \mathrm{ZnSe}(4 \mathrm{~nm})$ multilayer (c), measured under excitation with three laser lines denoted in the figures (in $\mathrm{nm}$ ).
The selection rule for zinc-blende crystal structures shows [10] that only LO modes are allowed when the incident laser beam is perpendicular to the (100) planes of a single crystal and only transverse optical TO modes are allowed when the incident laser beam is parallel to the (110) planes. Appearance of a weak TO band in the Raman spectra indicates that most likely crystal grains in the $1 \mu \mathrm{m}$ thick film are randomly oriented.

Figures 1b and 1c show Raman spectra of a ZnSe layer with a thickness of $30 \mathrm{~nm}$ and $\mathrm{SiO}_{x}(4 \mathrm{~nm}) / \mathrm{ZnSe}(4 \mathrm{~nm})$ multilayer, respectively. Both samples were deposited on c-Si substrate and the observed strong narrow band peaked at $521 \mathrm{~cm}^{-1}$ is due to scattering from the substrate. The $1 \mathrm{LO} \mathrm{ZnSe}$ band intensity increases with decreasing excitation wavelength and series of 4 peaks are seen only in the spectra excited by $457.9 \mathrm{~nm}$ line. Such behaviour of Raman spectra allows assumption that the bandgap energy of the $30 \mathrm{~nm}$ layer and the ZnSe layers in $\mathrm{ML}$ is higher than the energy of the $457.9 \mathrm{~nm}$ line, as expected due to the low ZnSe film thickness. Indeed, the characterization of $\mathrm{SiO}_{x} / \mathrm{ZnSe}$ MLs (with various thicknesses between 2 and $10 \mathrm{~nm}$ ) by Raman spectroscopy using the $442 \mathrm{~nm}$ line has shown [6] resonant behaviour in the Raman spectra. It has been related to size-induced change of the bandgap energy with the layer thickness and indicated that optical band gap of the layers thinner than $7 \mathrm{~nm}$ is $\geq 2.8 \mathrm{eV}$.

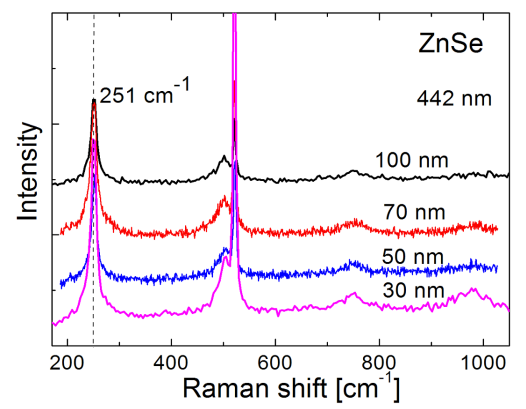

Fig. 2. Raman spectra of a series of ZnSe single layers with four different thicknesses (denoted in the figure) measured using the $442 \mathrm{~nm}$ laser line.

Figure 2 depicts Raman spectra of a series of ZnSe single layer having thickness between 30 and $100 \mathrm{~nm}$ measured using the $442 \mathrm{~nm}$ laser line. The intensity of the $1 \mathrm{LO}$ band of the $30 \mathrm{~nm}$ layer is significantly higher than those of the other samples which can be related to the existence of crystal grains with $E_{\mathrm{g}}^{0} \approx 2.8 \mathrm{eV}$, in which carrier confinement takes place. The size of such grains should be $\leq 10 \mathrm{~nm}$. Therefore, the apparent grain size of the nanocrystals resolved by the atomic force microscopy (AFM) [11], which is in the range from 25 to $30 \mathrm{~nm}$, is considerably larger than the real one. The Raman result implies that ZnSe layers thinner than $50 \mathrm{~nm}$ could show a good chemical sensitivity due to the small grain size.

The AFM results obtained on the studied ZnSe have shown [11] that as-deposited films having thickness in the 
range $30-100 \mathrm{~nm}$ are homogeneous and possess smooth surface. However, after 60 days relaxation in air at room temperature they showed pits on the surface and a relative high root mean square (rms) surface roughness of $2-4 \mathrm{~nm}$ for a length scale of $500 \mathrm{~nm}$. Therefore it has been assumed that as-deposited films are highly strained and the strain relaxation with time creates pits and increases the surface roughness. Besides, optical transmission measurements on relaxed films have revealed an energy dependence of the absorption coefficient typical for amorphous materials. Since both the AFM and the Raman scattering data have shown presence of nanocrystals, it can be concluded that the layers contain two phases, amorphous and crystalline and the amount of the crystalline phase decreases with decreasing thickness. Moreover, the line width of the $1 \mathrm{LO}$ band of $\approx 14-15 \mathrm{~cm}^{-1}$ is much greater than the one determined for $\mathrm{ZnSe}$ single crystal which amounts $\approx 6 \mathrm{~cm}^{-1}$. It was shown [10] that strain in ZnSe thin films does not appreciably affect their Raman spectra. Hence the significantly larger line width observed in this study is most likely due to both existence of amorphous phase and some lattice distortion in the crystalline grains due to their random orientation.

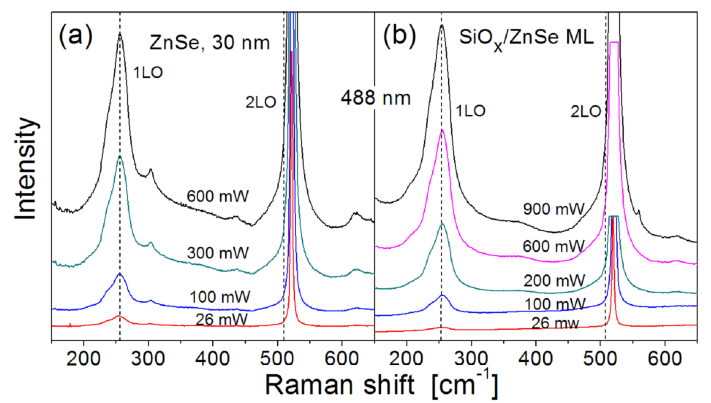

Fig. 3. Micro-Raman spectra of a $30 \mathrm{~nm}$ thick ZnSe single layer (a) and a $\mathrm{SiO}_{x}(4 \mathrm{~nm}) / \mathrm{ZnSe}(4 \mathrm{~nm}) \mathrm{ML}(\mathrm{b})$ measured with various output laser powers.

Figures 3a and 3b show micro-Raman spectra of both ZnSe single layers and ZnSe layers in MLs measured with increased laser power density of the $488 \mathrm{~nm}$ line and long accumulation time $(60 \mathrm{~min})$. The right dashed vertical lines in both figures mark the position of the 2LO band whose intensity should increase with increasing the volume of the crystalline phase. Indeed, the asymmetric shape of the Raman feature at $521 \mathrm{~cm}^{-1}$ assigned to TO band from c-Si for high output beam power $(\approx 300 \mathrm{~mW})$ can be related to the appearance of the $2 \mathrm{LO}$ band of ZnSe. This observation indicates that sample exposure to laser beam irradiation increases the crystalline portion in both kinds of samples. However, further studies on large sample area are necessary to make quantitative estimation of this effect.

\section{Conclusions}

Raman scattering has been applied in order to make structural characterization of thin and ultra thin $\mathrm{ZnSe}$ layers deposited at room substrate temperature using step-by-step thermal vacuum evaporation. The measurements performed under excitation with three different $\mathrm{Ar}^{+}$laser lines have shown that independently on the layer thickness the 1LO band intensity shows a maximum value when excited with the $457.9 \mathrm{~nm}$ line. The energy of this line is very close to the optical band gap of the $1 \mu \mathrm{m}$ layer and for this particular layer thickness resonant Raman scattering has been gained. The measurements carried out with the $442 \mathrm{~nm}$ line have indicated that its energy is closer to the optical band gap of the crystalline grains in the thinnest single layers and the ZnSe layers in MLs than the energy of the $457.9 \mathrm{~nm}$ line. This observation has indicated carrier confinement and implies that the grain size in these layers is $\approx 10 \mathrm{~nm}$. Large line width $\left(\approx 15 \mathrm{~cm}^{-1}\right)$ of the $1 \mathrm{LO}$ band observed in the spectra of all samples has been related to the existence of amorphous phase and some lattice distortion in the crystalline grains in the layers thinner than $100 \mathrm{~nm}$. The ZnSe film crystallinity has been improved with irradiation of the samples by high laser beam power $(\geq 300 \mathrm{~mW})$.

\section{Acknowledgments}

This work is supported by the Serbian Ministry of Science and Technological Development under the project No. 141047 and bilateral project between BAS and SASA.

\section{References}

[1] H. Shoji, Y. Nakata, K. Mukai, Y. Sugiyama, M. Sugawara, N. Yokoyama, H. Ishikawa Electron. Lett. 32, 2023 (1996).

[2] P. Reiss, G. Quemard, S. Carayon, J. Bleuse, F. Chandezon, A. Pron, Mat. Chem. Phys. 84, 103 (2004).

[3] D.S. Patil, D.K. Gautam, Physica B 344, 140 (2004).

[4] E. Guziewicz, M. Godlewski, K. Kopalko, E. Lusakowska, E. Dynowska, M. Guziewicz, M.M. Godlewski, M. Phillips, Thin Solid Films 446, 172 (2004).

[5] D. Nesheva, Z. Aneva, S. Reynolds, C. Main, A.G. Fizgerald, J. Optoel. Adv. Mater. 8, 2120 (2006).

[6] M.J. Šcepanovic, M. Grujić-Brojčin, I. Bineva, D. Nesheva, Z. Aneva, Z. Levi, Z.V. Popović, J. Optoel. Adv. Mater. 9, 178 (2007).

[7] D. Nesheva, in: Handbook of Surfaces and Interfaces of Materials, Ed. H.S. Nalwa, Vol. 3, Academic Press, San Diego 2001, p. 239.

[8] M. Cardona, G. Guntherodt, Light Scattering in Solids, Springer, New York 1982, p. 191.

[9] D. Nesheva, Z. Aneva, Z. Levi, N. Vuchkov, K. Temelkov, I. Bineva, Nanosc. Nanotechnol. 7, 83 (2007).

[10] Y. Kanemitsu, A. Yamamoto, Hitoshi Matsue, Y. Masumoto, Sh. Yamaga, A. Yoshikawa, Appl. Phys. Lett. 60, 16 (1992).

[11] D. Nesheva, M.J. Šcepanovic, Z. Levi, S. Askrabic, Z. Aneva, A. Petrova, Z.V. Popović, J. Optoel. Adv. Mater., accepted for publication. 